\title{
Physical Internet-Enabled Manufacturing Execution System for Intelligent Workshop Production
}

\author{
Ray Y. Zhong ${ }^{* 1}$, Huajun Gong ${ }^{1}$, Chen $\mathrm{Xu}^{2}$ and Shaoping $\mathrm{Lu}^{3}$ \\ ${ }^{1}$ College of Information Engineering, Shenzhen University, Shenzhen, China \\ ${ }^{2}$ Institute of Intelligent Computing Science, Shenzhen University, China \\ ${ }^{3}$ Department of Transportation Economics and Logistics Management, Shenzhen \\ University, China \\ *zhongzry@gmail.com
}

\begin{abstract}
A Physical Internet-enabled Manufacturing Executive System (PIMES) is proposed for intelligent workshop production. PIMES uses Radio Frequency Identification (RFID), 433MHZ wireless communication, and Physical Internet concept to create a ubiquitous production environment where real-time data could be collected and then converted into dynamic feedback factors sent back to the system. PIMES includes several services such as Production Decision Service, Communication Control Service, Visibility Service, and Interface Service which are designed and developed by making full use of the serviceoriented architecture (SOA) and Cloud technology. With the assistance of these services, PIMES is able to achieve several outperformances upon traditional production systems like paperless operations, real-time decision-making, automatic statistics and reporting, as well as visualized manufacturing control and management. After the implementation of PIMES, workshop production of a case enterprise is streamlined and optimized by making full use of the collected data to support the workshop production and logistics.
\end{abstract}

Keywords: RFID, Physical Internet, Intelligent Workshop, MES, Production

\section{Introduction}

Physical Internet (PI) is an open global logistics system which is based on physical, digital, and operational interconnectivity, through encapsulation, interfaces and protocols so as to replace current logistical models [1-2]. The project currently has funding from the National Science Foundation as well as contributions from MHIA and CICMHE [3]. After the great success of applying PI in the global supply chain, this concept has been widely implemented so as to upgrade the current supply chain into a smart level [4].

Mass-Customized (MC) production refers to provide customized products or services through flexible processes in great myriad and low costs [5]. In recently, customer driven manufacturing plays a primary role in MC production, which a systemic methodology involving all aspects of product sale, development, production and delivering, full-circle from customer's options [6]. Manufacturing in workshop is one of bottlenecks in MC enterprises [7-8]. It greatly affects the production cycle because great many challenges are faced by MC workshop. First of all, it is labor-costing and material-costing as the reason of lack of estimation about capacity in the workshop. The estimation should be based on large amount data accumulation from MC plants. Unfortunately, those data are recorded in papers. It is fatal for an enterprise when the main documents are missing. Secondly, information communication is actually depended on phone calls and e-mail. Most information about manufacturing is delayed when some changes occurred in customers [9]. Confusions are also happened to workshops by phone and e-mail when some detail

*Corresponding Author 
parameters should be changed or revised. Then, logistics in MC workshop is out of control because there is no efficient way to tracking materials. Finally, workers do not know what to do, how to do, where can fetch materials and how much they would be paid. Workers in workshops lose enthusiasm to fulfill and compete with others as tasks are distributed [10]. As a result, manufacturing production in MC workshop is greatly confined. Those challenges in MC workshop deeply obstruct exploration of machinery capacity and manufacturing efficiency.

In order to address these challenges, IT-based systems are proposed to be the most suitable method in MC workshop [11]. Some information systems focus on addressing the upper level decision-makings such as planning and scheduling like ERP (Enterprise Resources Planning) system. Such systems lack of capacity of workshop management. Some are auxiliary for enterprise technology design and agile response to customer management such as CAD [12]. When there are some urgent changes happened to customers, it is difficult for those systems to deliver information to workers in plants directly.

This paper introduces a Physical Internet-enabled Manufacturing Executive System (PIMES) for MC workshop. PIMES based on RFID for real-time data collection is designed and developed to tackle the above challenges. First, RFID devices like readers and tags are deployed on various manufacturing objects to create a ubiquitous production environment. Second, a wireless communication network with $433 \mathrm{MHz}$ standard is used for transmitting the data within Intranet or Internet. Third, a SOA-based architecture is designed and established for enabling various services to plug into the system framework so that various end-users are able to use these services to facilitate their daily operations.

The remainder of this paper is arranged as follows. Section 2 presents the PIMES in terms of motivating case and PI-enabled intelligent workshop. Section 3 reports on the design and development considerations from hardware and software perspectives. Section 4 demonstrates a case study from a heavy-duty machinery manufacturer to show the feasibility and practicality of PIMES to streamline the workshop production. Section 5 concludes this paper by providing future research directions.

\section{Physical Internet-Enabled Manufacturing Executive System}

\subsection{Motivating Case}

The motivating case comes from a distributed mass-customized production enterprise, which manufactures a ceramic and other large-scale integration of mechanical, electrical and mechanical equipment. Figure 1 demonstrates the production processes. There are two main factories which locate in different area with a distance about $10 \mathrm{KM}$. Each factory owns several workshops which are equipped with several machines. With the customized products from customers, some components are outsourced, and some main components are made in the workshops. Manufacturing production is organized by Production Department, Quality Department, Machinery Department, Technology Department and Assistant Department. Assistant Department mainly deals with some special processes such as the processes cannot be accomplished in the enterprise. After all the components are finished, they will be sent to the assembly workshop. 


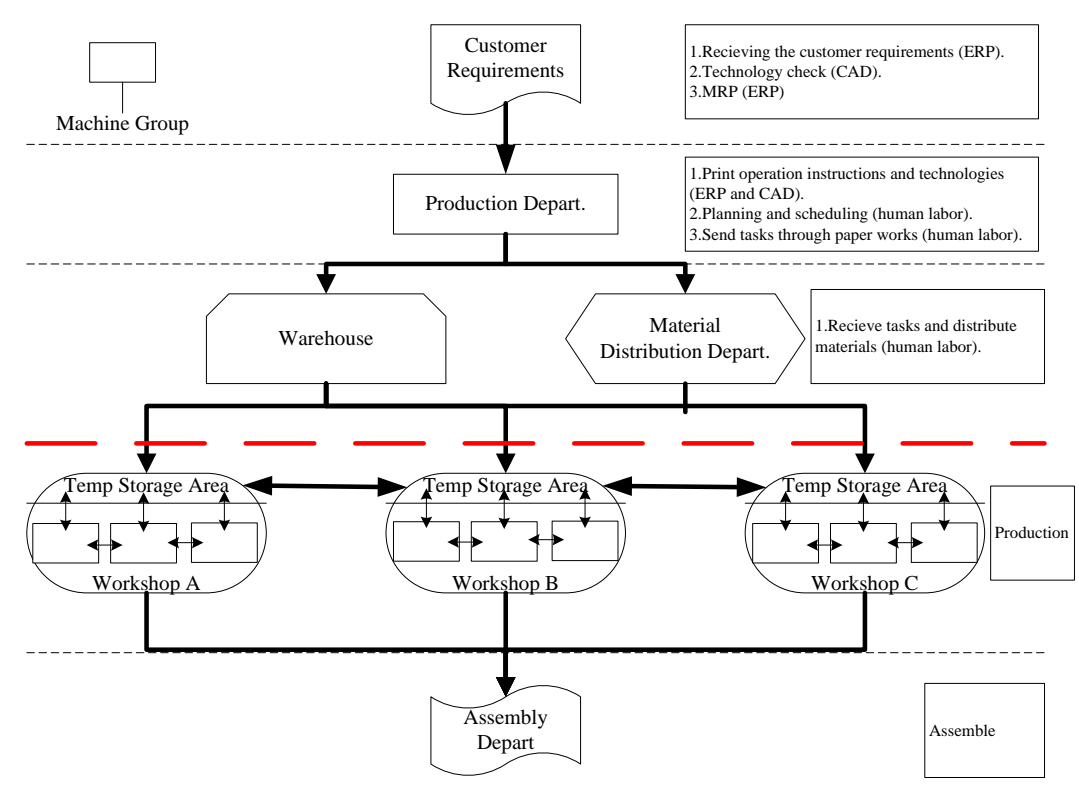

Figure 1. Production Processes in the Case Company

Workshops production is very hard to control since there are frequent varieties of order inserting, revising, and cancelling. Despite the enterprise has set up SAP ERP, PDM, OA and CAPP, they are incapable of controlling workshop manufacturing. As a result, incoherence of plans and executions is challengeable. Several detailed issues are listed:

(1) Production capacity is ambiguous and hard to measure without the data from workshops.

(2) Data in workshop are managed by papers manually.

(3) Large myriad of varieties are occurred in workshop resulting in re-planning and re-scheduling frequently.

(4) Performance management is difficult to establish due to lack of production data.

(5) Production efficiencies are confined since no optimal approaches are adopted in the manufacturing workshop.

In order to track these challenges, PIMES uses RFID and $433 \mathrm{MHz}$ wireless communication to collect the real-time data in the workshop. RFID reader is designed and developed as IDT (Intelligent Data Terminal) so as to capture the realtime production data and enables the operators to facilitate their daily operations.

\subsection{PI-Enabled Intelligent Workshop}

In the workshop, machineries are categorized into different groups. Each IDT owns an identical address associated with a machine and the binding relationship between IDTs and machineries could be configured in a service. IDTs identify not only machines, but also differentiate workers and materials which are carrying tags. Each worker has a RFID-enabled staff card as ID card [13]. This method is based on an integration of workers, materials, IDTs and machines together by using RFID technology. Some critical information could be set in PIMES such as how many types of machinery would be operated by a worker, which group the machine belongs to, which IDT associates with the machine, etc. In order to track logistics, each batch of materials owns a tag which is used to identify materials and it could be recycled. 


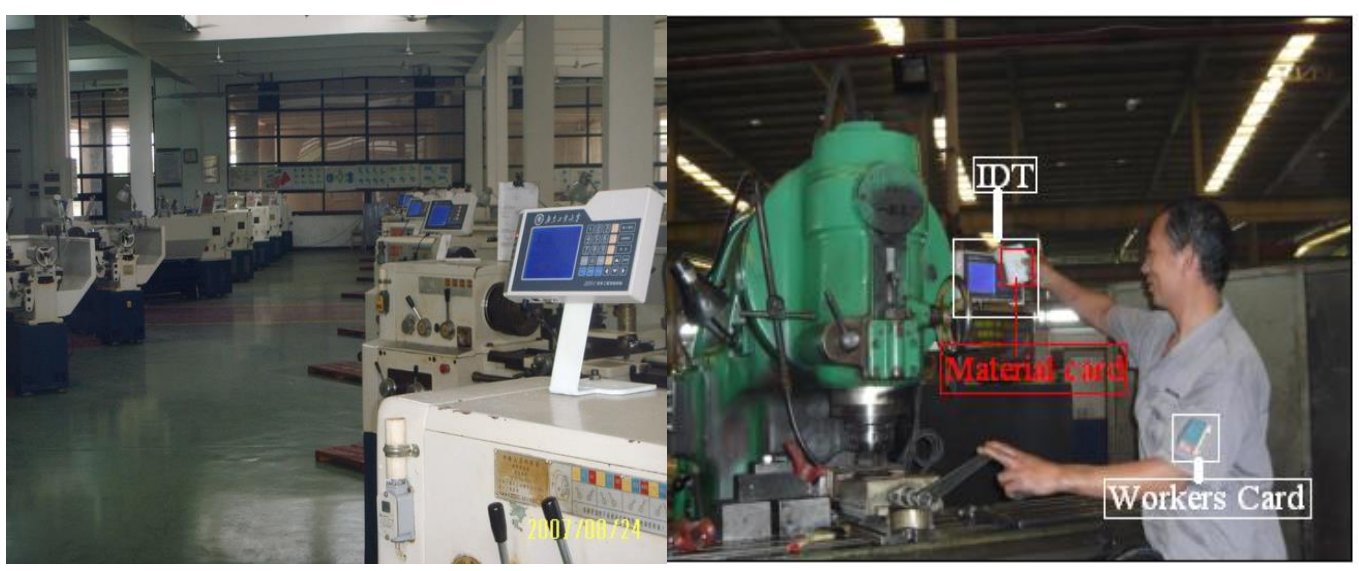

Figure 2. IDT Deploy Methodology

2.2.1. Deployment of IDTs: As to facilitate the manufacturing flows according to processes and logistics, each machine and buffer is taken to be a node in manufacturing network. Raw-material and finished products are defined as begin and end in the network respectively. IDTs should be deployed in each node for collecting the critical information. Each machine is equipped by an IDT for displaying the instructions and collect data. However there are some sophisticated machines unavailable to install IDTs. In such cases, IDTs are installed in the wall or ground near the machines. Figure 2 shows the real-life PI-enabled workshop environment with deployed IDTs. It could be learned from practice lessons that locations installed with IDTs should be as convenient as possible for the workers' daily operations.

Additionally, in order to trace logistics. IDTs are also deployed in the buffers [14]. When materials are delivered in or out of the buffers, some operations will be carried on these devices such as confirm operation, etc. Meanwhile, basic data about processes and logistics is captured and collected so that real-time statuses are obtained.

2.2.2. Deployment of Tags: High frequency (HF) is chosen in the PIMES considering the environment in MC workshop which are full of oil pollution. Each batch of materials is attached by a HF tag and delivered from the beginning of first node to the last one, including all the logistics processes. There are two approaches to attach the tags. One is at the first processing stage where a batch of materials is finished, PIMES informs the operators to attach a tag via IDT. Another is at the warehouse raw-material preparing area, where tags could deployed as convenient as necessary [15]. Both approaches should establish some temp deposit where materials are located during production. Some operations should be amenable through IDTs such as register operator, handing operations etc so as to tracking logistics.

After the deployment, tags combine with some basic information such as job instructions, technical parameters and figures, machine attributions and quality check items etc. These data are stored in the database with index-record-storage (IRS) methodology which uses index form and layer-distributed association for linking all the associated data. This storage methodology can reduce complexity in both data and database [16]. And another methodology used is data cache used temporary unit to keep large bulk of data under dynamic multi-compression with multi-layers. Through unique tag code searching in the database, all the information can be transferred correctly by using the wireless communication network. 
2.2.3. Deployment of Communication Network: Efficient network is primary for MC workshops to transfer the captured data reliably and securely. PIMES uses $433 \mathrm{MHz}$ wireless communication for this purpose. A device called Base Station (BS) is designed and developed for enabling the wireless network which plays an important role in the communication. BS collects the data sensed by IDTs, and then sends them to a Work Station (WS) via cable. Figure 3 presents the communication network deployed in the workshop.

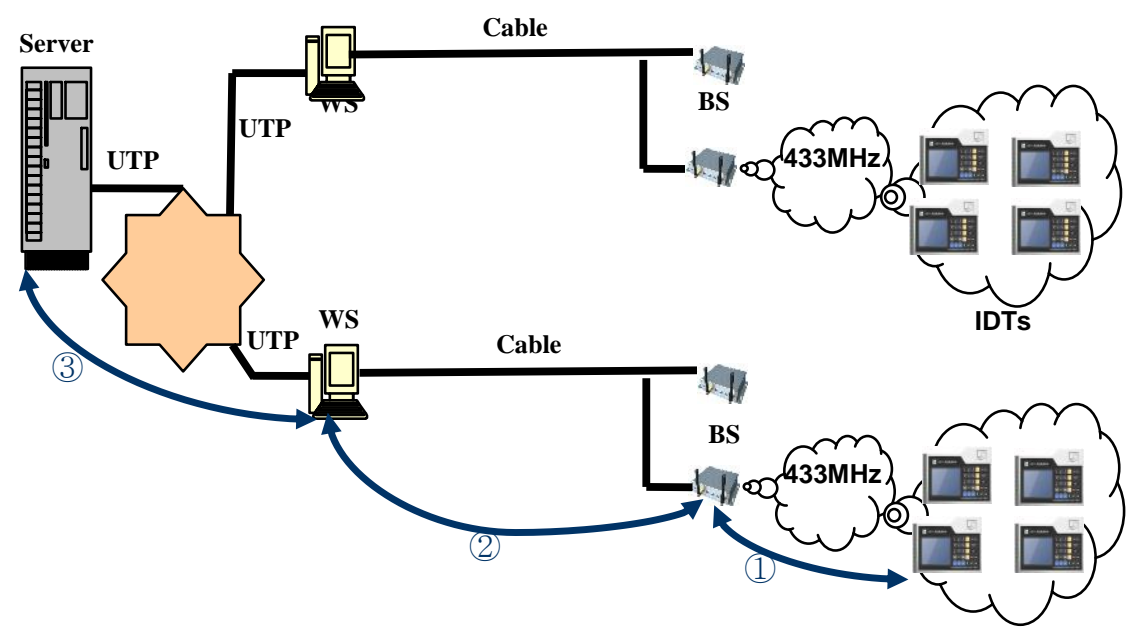

Figure 3. 433MHz Based Wireless Communication Network

From the above Figure 3, data are communicated with the wireless network following three major steps. (1): When the IDTs read a tag or some operations occurred; IDTs will send the sensed data to a BS through $433 \mathrm{MHz}$ wireless communication standard. (2): A BS receives these data and sends them to a WS through a cable. (3): A service in WS mainly analyzes the data and then sends to a server via LAN. Finally, server receives the data and works out corresponding results. This network is an easy-implement approach for MC workshop to collect and transfer the data considering the cost benefit analysis. As a result, small and medium sized enterprises can afford such solutions in their manufacturing workshops. In this network, random parameters are proposed to address the collisions between IDTs.

After the deployments, all the manufacturing objects are identified and an intelligent manufacturing environment is created. Within this environment, all the production data are captured and collected when the objects interconnected to each other. Thus, PIMES is able to make associated decisions by making full use of the data and such decisions could be sent back to corresponding parties on a real-time basis. By predefining the production logic and logistics workflows, the manufacturing operations and logistics behaviors could be streamlined within this environment. 


\section{Design and Development Considerations}

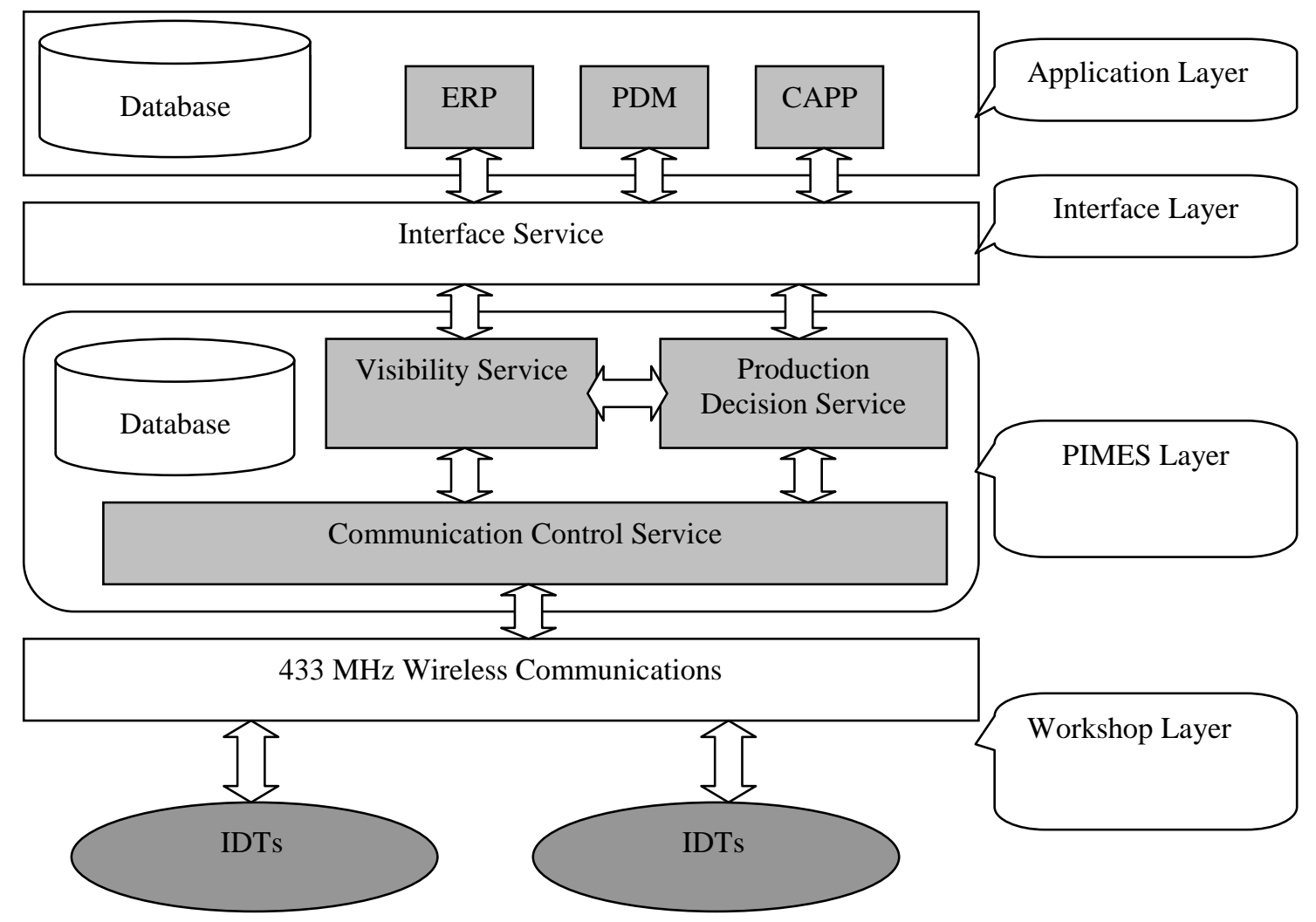

Figure 4. Overview of PIMES

For easing the design and development, PIMES is divided into four layers: Workshop Layer, RRMES Layer, Interface Layer and Application Layer as shown in Figure 4. Workshop Layer mainly contains IDTs, BSs, $433 \mathrm{MHz}$ wireless network and other devices which are located in the workshop. They are the main hardware in this system. PIMES Layer contains several major services: Communication Control Service, Production Decision Service, and Visibility Service. They accomplish main function in cooperation of hardware. Interface Layer includes a service designed as a middleware which can communicate with other information systems through normative data structure predefined. Thus, the timed and real-time data intercommunication between each system could be realized. The application layer is consisted by some management systems and auxiliary information systems, such as ERP, CAPP, PDM, CRM, OA, etc.

In PIMES, all data are delivered from one standard interface to other application systems. Those systems are able to use the real-time data from workshops for advanced decision-makings. To this end, hardware and software are designed.

\subsection{Design of Hardware}

In PIMES, taking the communication network system as an example, it is a platform which contains IDT, BS, cable, PCI cards, WS, UTP and server. There are two crucial devices in this system. One is IDT and another is BS. 
3.1.1. Intelligent Data Terminal (IDT): IDT is an input/output device which is deployed in the workshops. It mainly involves seven main components, which are RFID, network, data input, state display, data display, central process unit, and memory. Data structure in IDTs is defined as follows:

Start (1) address (4) CID (1) STEP (1) INFOLEN (4) XXXX (information contents) CHECK (4) End (1). Start: beginning of character string, (1)-represents the length of 1 Byte; address: the address of IDT with the length of 4 Byte; CID: Current window identity uses 1 Byte; STEP: the next CID of window; INFOLEN: context length of information; CHECK: sum ASCII of all characters then 'and' the sum with FFFF; end: flag of the end.

For example: $\mathrm{CID}=0, \mathrm{STEP}=0$ represents an identity code of one window. The string “\#1'0105'\#\$A\#\$A'0000019A'\#2" could be interpreted as: \#1: data head, \#2: data tail, '0105': IDT address, '\#\$A\#\$A': stands for $\mathrm{CID}=0$, STEP=0, '0000': information contents, '019A': check code. IDT sends such string to BS. Then WS receives and analyzes it. If the required window code is $\mathrm{CID}=3, \mathrm{STEP}=0, \mathrm{CID}$ and STEP are converted into '\#\$D\#\$A'.

3.1.2. Base Station: BS (Base Station) is another communication device in PIMES. It is a bridge between WS and IDTs. It contains five main parts: network control, data process, memory, MCU, and send/receive unit. BS communicates with IDTs through $433 \mathrm{MHz}$ standards. Each BS occupies one frequency with $50 \mathrm{KHz}$ as bandwidth so as to void interference. Each BS manages several IDTs whose quantity could be configured. In order to communicate with IDTs, BS turns inquiry to each IDT continuously. If there are requirements occurred, BS will process accordingly, then goes on turning.

\subsection{Software Design}

PIMES software contains four primary services: Communication Control Service, Production Decision Service, Visibility Service, and Interface Service. In order to tackle the limitation of the network bandwidth, Production Decision Service and Communication Control Service are located in WS and the other two are located in the server. Each service could share data according to the established protocol.

Production Decision Service mainly executes workshop management decisions like manufacturing planning and scheduling. It manages the production in MC workshop, including task inserting, deleting, halting and adjusting. Communication Control Service manages all data transferring. It also controls the IDTs and communication hardware devices. Visibility Service is a frequently used tool for all the users. It provides important data view and processes monitoring results. Interface Service is a bridge between PIMES and other systems. Its main function is intercommunicating with upper systems via real-time data delivering.

\subsection{Real-Time Methodology}

Real-time manufacturing in MC workshop is important to meet the stochastic customer requirements. In PIMES, there are three real-time methodologies. First of all, a real-time information system platform is proposed by structuring the real-time data collectors and communication approaches. Secondly, a real-time interface is used for data transferring with other systems. Finally, a real-time decision-making party with the data is enabled in the Production Decision Service for making suitable manufacturing optimizations. In this section, real-time interface is detailed illustrated.

The real-time interface is based on middleware mechanism, which defines a set of protocol. This interface provides data sharing and intercommunication service with 
other systems. It contains two main queues, one is real-time queue, and another is timed queue. Real-time queue uses SOCKET to deal with real-time requisitions. Timed queue could be configured by parameters to achieve timely processing. Both of real-time and timed queues can directly call the function modules which are encapsulated in DLLs.

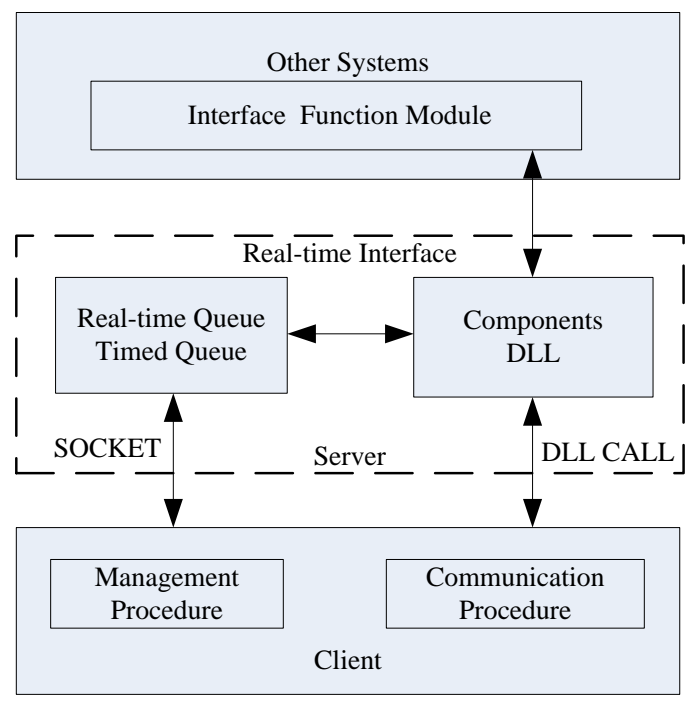

Figure 5. Principle of Real-Time Interface

Figure 5 indicates the real-time principle in the interface. The interface is workable after some configurations including data sources, function module, execution time etc. After configuration, Interface Service can execute automatically according to the predefined protocol and working logic. The protocol and data structure are unified criterion for the systems which need intercommunicate to each other.

\section{Case Study}

The case study comes from a typical MC enterprise which focuses on MC products from world-wide customers. It specializes in manufacturing the ceramic, stone, wall material, energy saving and environmental protection, and other largescale integration of mechanical and technical devices. The company produces such series products according to the requirements from customers. Because of the complexities and weak management level in workshop level, products costs are super high. Thus, a workshop level IT system is necessary and PIMES is designed and developed for this case.

PIMES plays an important role in the whole production management cycle from start of raw-materials to finished products in workshop level. Great improvements of production management are achieved after implementing PIMES. In the first place, decision-making hierarchies are profoundly changed according to the PIMES infrastructure. The following Figure 6 shows how PIMES improves the decisionmaking after program architecture changed. The new program architecture could suit the complex manufacturing environment of tracking, monitoring, collecting, planning, scheduling, and execution. 


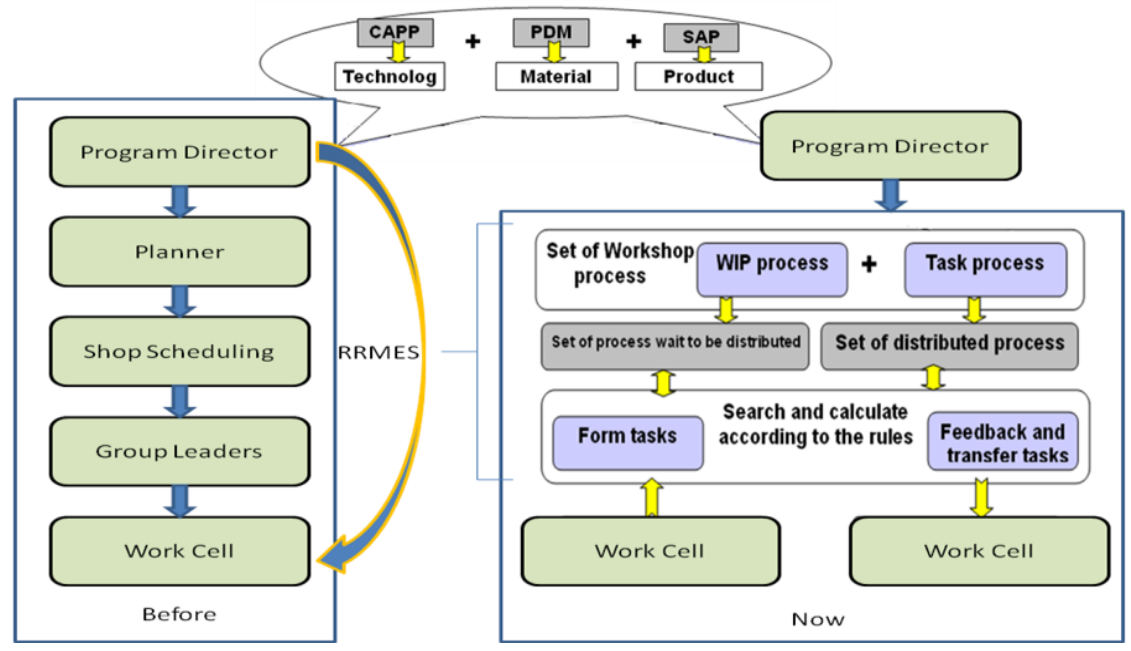

Figure 6. Decision-Making Hierarchy in PIMES

Secondly, after all the IDTs and BSs are deployed in the MC workshop, production and logistics operations are real-time controlled so that the corresponding data are captured and collected. PIMES thus manages all manufacturing sites and buffers via IDTs. The following Figure 7 shows how the manufacturing in MC workshops reengineered and rationalized by the PIMES. And some improvements are also showed comparing with the previous production system based on manual and paper work.
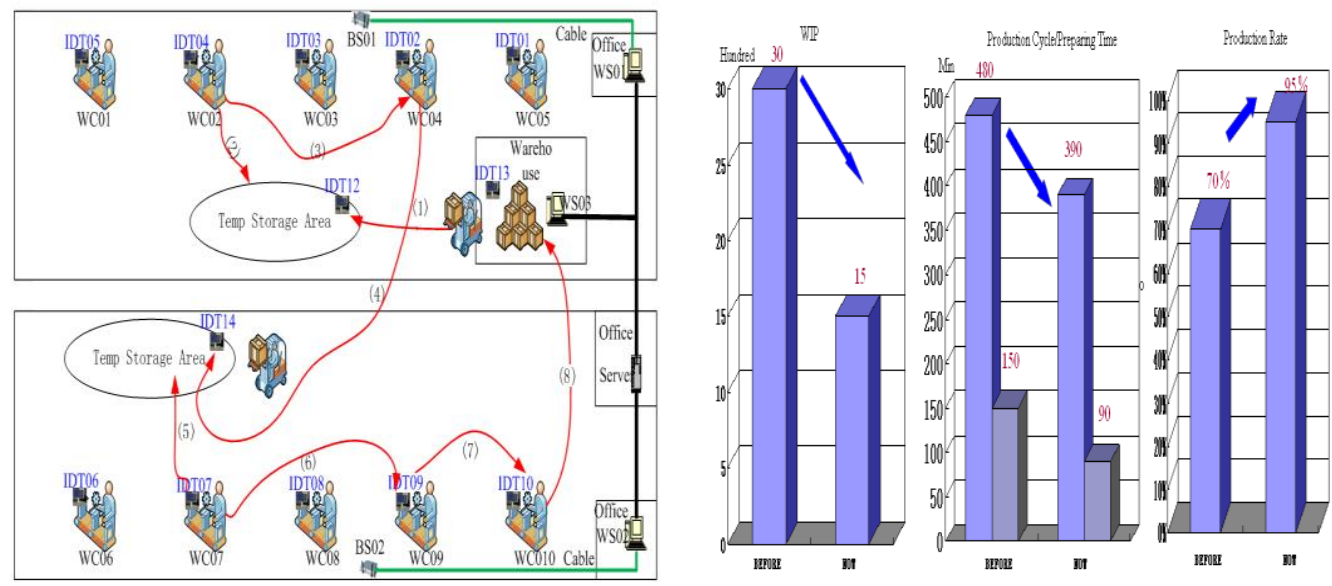

Figure 7. Reengineered Manufacturing and Improvements in MC Workshop

Finally, PIMES has been implemented after six months in the MC workshops from the case company. After one month trail, data storage achieved over $600 \mathrm{M}$. these data include quality check, worker behaviors, logistics data etc. The myriad of data storage indicates how much production data in MC workshop and they are invaluable for supporting advanced production decision-makings. Table 1 shows the statistics improvement from the collected data. 
Table 1. Quantitative Improvements

\begin{tabular}{|c|c|}
\hline Improved Items & Percentage \\
\hline Shorten manufacturing cycle & $>12 \%$ \\
\hline Reduce or eliminate data entry & $30 \%$ \\
\hline Reduce WIP & $18 \%$ \\
\hline Reduce paper work & $57 \%$ \\
\hline Shorten lead time & $>22 \%$ \\
\hline Improve product quality & $>12 \%$ \\
\hline Reduce waste of write operation & $45 \%$ \\
\hline Paperless work & $>90 \%$ \\
\hline Information in-time & $96 \%$ \\
\hline
\end{tabular}

After the implementation of PIMES, from Table 1, workshop production of this enterprise is streamlined and optimized by making full use of the captured data to support the workshop production and logistics. Staffs in the enterprise comment the PIMES as "PIMES is a flexible system which is able to respond to varieties and provides agile manufacturing which we have dreamed for a long time. That is most important for us." One employee in the production department cited when he used this system "It allows us to meet higher sale volumes without increasing cost. Supervisors empower to make decisions timely with reliable information from manufacturing workshops. Specifically, this system provides production planning and scheduling through inserting orders and lock-in schedule dates which is more reasonable." A person in the quality department says "Large quantity of quality data is collected via IDTs. As a result, it is easy to track and monitor quality issues."

\section{Conclusions}

This paper discusses an information system named PIMES for MC workshop. PIMES relies substantially on RFID and wireless communication networks for the collection and synchronization of real-time data. The emphasis of this paper is placed on reveal how to achieve real-time manufacturing in MC workshop by designing and developing a hardware platform including RFID reader and tags deployment, communication network, as well as software perspectives.

After practical application from a case study, PIMES is capable of production management, quality monitoring, real-time data collection, and equipment monitoring. Meanwhile, this paper illustrates the integration of RFID, $433 \mathrm{MHz}$ wireless communicating and real-time methodologies to deal with the challenges in typical MC workshops. PIMES has been extended in large-machinery assemble enterprises, glass manufacturing and mold and die industry, and even the engineering training center in high school [13]. It accomplishes excellent performance in these fields.

In addition, two limitations are needed to be improved. First of all, communication stability is depended heavily on BS (Base Station). BS occupies a channel frequency. IDTs controlled by this BS are incapable to communicate with other BS because the disparate channel frequency. The other limitation is aprotic communication channel between BS and IDTs. IDTs cannot choose the nearest BSs to communicate automatically. Changes of this communication mode should be reconfigured by professional workers. 
Further research and improvement are also necessary if PIMES is of great merits to promote. First, as the introduction of CEP (Complex Event Processing) which could deal with operation efficient problems and discover more actionable information, CEP application in PIMES is a possible further research direction to improve the efficiency. CEP could be important in enterprises information systems to construct decoupled, and many to many communication systems. Second, the usage of Oracle database is needed to meet the increasing volume of data. Third, PIMES is lack of efficient auto-data analysis and data mining tools. Decisionmakings are always needed by the aide of officials. Thus, data analytic models could be further investigated. Finally, B/S architecture is popular as its brilliant performance through networks. Future works combined with B/S and RFID are in schedule especially for MC workshop through cloud technologies.

\section{Acknowledgments}

This work is supported by National Natural Science Foundation of China (Grant No. 51405307, 61473093, 61401283) and Guangdong High Education Institution project (2013CXZDC008).

\section{References}

[1] B. Montreuil, "Toward a Physical Internet: meeting the global logistics sustainability grand challenge," Logistics Research, vol. 3, (2011), pp. 71-87.

[2] B. Montreuil, "Physical Internet Manifesto V1. 7: globally transforming the way physical objects are handled, moved, stored, realized, supplied and used," Québec, CA, (2010).

[3] R. Sample, "Rethinking the global supply chain," Science, vol. 1100, (2014), pp. 1103.

[4] B. Montreuil, R. D. Meller and E. Ballot, "Physical Internet Foundations", in Service Orientation in Holonic and Multi Agent Manufacturing and Robotics, ed: Springer, (2013), pp. 151-166.

[5] G. D. Silveira, D. Borenstein and F. S. Fogliatto, "Mass customization: Literature review and research directions", International journal of production economics, vol. 72, (2001), pp. 1-13.

[6] R. Y. Zhong, Q. Dai, T. Qu, G. Hu and G. Q. Huang, "RFID-enabled real-time manufacturing execution system for mass-customization production," Robotics and Computer-Integrated Manufacturing, vol. 29, (2013), pp. 283-292.

[7] M. L. Wang, T. Qu, R. Y. Zhong, Q. Y. Dai, X. W. Zhang and J. B. He, "A radio frequency identification-enabled real-time manufacturing execution system for one-of-a-kind production manufacturing: a case study in mould industry", International Journal of Computer Integrated Manufacturing, vol. 25, (2012), pp. 20-34.

[8] Q. Y. Dai, R. Y. Zhong, G. Q. Huang, T. Qu, T. Zhang and T. Y. Luo, "Radio frequency identificationenabled real-time manufacturing execution system: a case study in an automotive part manufacturer," International Journal of Computer Integrated Manufacturing, vol. 25, (2012), pp. 51-65.

[9] R. Y. Zhong, G. Q. Huang, Q. Y. Dai and T. Zhang, "Mining SOTs and Dispatching Rules from RFIDenabled Real-time Shopfloor Production Data," Journal of Intelligent Manufacturing, vol. 25, (2014), pp. 825-843.

[10] R. Y. Zhong, Q. Y. Dai, K. Zhou and X. B. Dai, "Design and Implementation of DMES Based on RFID", in 2nd International Conference on Anti-counterfeiting, Security and Identification, Guiyang, (2008) Aug. 20-23, pp. 475-477.

[11] F. S. Fogliatto, G. J. da Silveira and D. Borenstein, "The mass customization decade: An updated review of the literature", International Journal of Production Economics, vol. 138, (2012), pp. 14-25.

[12] M. M. Tseng and S. J. Hu, "Mass customization," CIRP Encyclopedia of Production Engineering, (2014), pp. 836-843.

[13] R. Y. Zhong and G. Q. Huang, "RFID-enabled Learning Supply Chain: A Smart Pedagogical Environment for TELD", International Journal of Engineering Education, vol. 30, (2014), pp. 471-482.

[14] R. Y. Zhong, G. Q. Huang, S. L. Lan, Q. Y. Dai, T. Zhang and C. Xu, "A two-level advanced production planning and scheduling model for RFID-enabled ubiquitous manufacturing", Advanced Engineering Informatics, http://dx.doi.org/10.1016/j.aei.2015.01.002, (2015).

[15] Q. Y. Dai, R. Y. Zhong, G. Q. Huang, T. Qu, T. Zhang and T. Y. Luo, "Radio frequency identificationenabled real-time manufacturing execution system: a case study in an automotive part manufacturer", International Journal of Computer Integrated Manufacturing, no. 25, (2012), pp. 51-65.

[16] R. Y. Zhong, G. Q. Huang, S. Lan, Q. Dai, X. Chen and T. Zhang, "A big data approach for logistics trajectory discovery from RFID-enabled production data", International Journal of Production Economics, no. 165, (2015), pp. 260-272. 


\section{Authors}

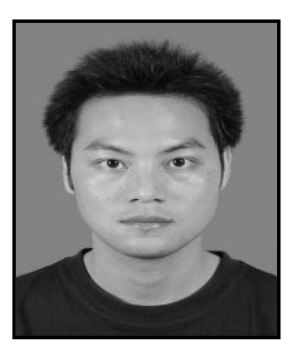

Ray Y. Zhong, he received the Ph.D. degree in Industrial and Manufacturing Systems Engineering from the University of Hong Kong, China, in 2013, M.S. degree in signal and information processing from Guangdong University of Technology, China, in 2009 and B.S. degree in mathematics and computer science and technology from Gannan Normal University, China, in 2004. His research interest includes Big Data in manufacturing and service, Advanced Planning and Scheduling, and RFID in Internet of Manufacturing Things. Dr. Zhong is member of LSCM HK, POMS, ASME, IIE, and IEEE. He was a recipient of the Best Conference Paper Award in the 2014 IEEE International Conference on Networking, Sensing and Control (ICNSC14) and Certificate of Merit in the Hong Kong U-21 RFID Awards 2011, GS1 HK. Dr. Zhong has published over 70 papers in reputable international journals and conferences with the total Google citation over 400.

Chen $\mathbf{X u}$, he is current a professor in Institute of Intelligent Computing Science, Shenzhen University, China. His research interests include intelligent computing, mathematics models and optimization algorithms. 